\title{
Building an Image-Based System to Automatically Score Psoriasis
}

\author{
David Delgado Gomez ${ }^{1}$, Jens Michael Carstensen ${ }^{1}$, Bjarne Ersbøll ${ }^{1}$, \\ Lone $\mathrm{Skov}^{2}$, and Bo Bang ${ }^{2}$ \\ 1 Image processing and computer vision, Informatics and Mathematical Modelling, \\ Richard Petersen Plads \\ +45 45253351, \\ 2800 Lyngby, Denmark \\ \{ddg, jmc, be\}@imm.dtu.dk \\ http://www.imm.dtu.dk \\ 2 Department of dermatology, Gentofte hospital \\ Niels Andersenvej 65, Denmark \\ $+4531651299$ \\ 2900 Hellerup,Denmark, \\ pbtoft@dadlnet.dk, BOBAN@gent of tehosp.kbhamt.dk
}

\begin{abstract}
Nowadays the medical tracking of dermatological diseases is imprecise. The main reason is the lack of suitable objective methods to evaluate the lesion. The severity of the disease is scored by doctors just through their visual examination. In this work, a system to take accurate images of dermatological lesions has been developed. Mathematical methods can be applied to these images to obtain values that summarize the lesion and help to track its evolution. The system is composed of two elements. A precise image acquisition equipment and a statistical procedure to extract the lesions from the images. The system is tested on patients with the dermatological disease psoriasis. Temporal series of images are taken for each patient and the lesions are automatically extracted. Results indicate that to the images obtained are a good source for obtaining derived variables to track the lesion.

Keywords: image acquisition, camera calibration, mixture distribution, discriminant analysis, E-M algorithm, psoriasis.
\end{abstract}

\section{Introduction}

Pharmaceutical companies constantly develop drugs to treat different diseases. One of their main interests is to get to know the effects of their products. To assess this, clinical trials involving several patients at many centers (hospitals) are conducted. However in the dermatological field there are few objective methods to evaluate the effects. Doctors visually assess the lesion and make scorings and journal notes of the condition. These notes and perhaps some photographs are presently the only memory of what the lesion looked like at the corresponding visit. Clearly, the scores are highly doctor dependent. Different doctors give different scores of the same patient because of differences in the doctor's perception. 
Moreover, the same doctor can score the same patient differently depending on the severity of the previous patient. Doctors tend to score a patient less severely when the previous one has a severe lesion. This all adds to the fact that in the dermatological field drug efficacy in many cases is not measured accurately.

The fast development in computer technology, that allows processing of huge amounts of information in a few seconds, together with the advances in image analysis during the last decade give an alternative to these rudimentary methods. Engström [1] observed the effect of a new enzymatic debrider observing the evolution of the lesion area and the lesion color. These measurements were obtained from digitized photographs analyzed with a computer. The obtained results pointed out the usefulness of image analysis to track the evolution of the lesion even when the system did not include good equipment for the acquisition of the image and it required user interaction to standardize the images.

Later, Hansen [2], developed an image system that included calibration for increasing the quality of the images. The system was used in animals but the possibility of being used in humans was mentioned. Deeper works related with color camera calibration were developed by Marszalec [3]. However, the problem of collecting standardized images that can be compared in time has not been fully solved. Gutenev [4](2001) shows two actual problems in the acquisition of the images: the specular reflection and missaligments.

In this work an image-based system to obtain suitable images that afterwards can be mathematically scored is proposed. The system performs two tasks. First, the equipment has been designed to obtain standardized, geometrically and spectrally corrected images in a reproducible way. These targets aim to guarantee that the images can be analyzed correctly and that images taken at different times can be compared. The second task is developed by a statistical procedure. Once the images have been taken, the system automatically separates the lesion from the healthy part. Statistical/pattern recognition tools can be used on these regions to obtain derived variables that describe both the areas and their relations.

\section{Image acquisition}

In order to take highly reproducible images a closed optical system is utilized. The system is made up of a 3-chip CCD camera, a halogen light source and an integrating sphere.

Figure 1 left shows a 3D computer image of the equipment. In this image, the position of the camera relative to the sphere can be observed. Figure 1 right displays the physical implementation of the equipment. The camera used in the equipment is a JAI CV-M90 3CCD RGB, with a Cosmicar/Pentax $25 \mathrm{~mm}$ lens.

The equipment is designed with the goal of producing completely diffuse light to avoid shadows and specular reflections. Specular reflections are produced when the source of light comes from above the object. Shadows, on the other hand, are produced if the source of light has a small angle to the object, and it comes from just one direction. To avoid both specular reflection and shadows, light has to 

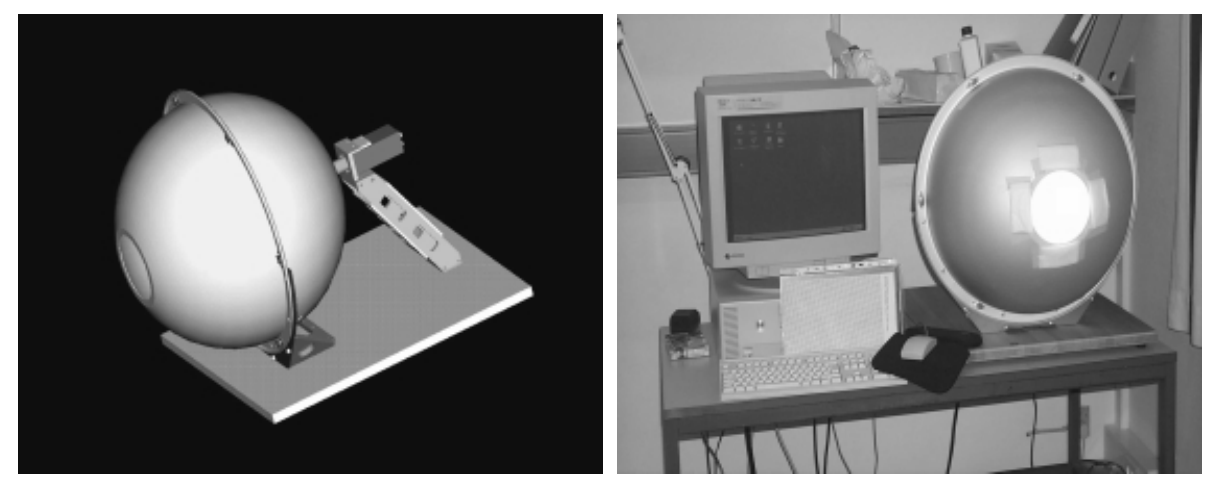

Fig. 1. Left:A 3D image of the equipment. Right: Equipment set in Gentofte hospital

come from all direction at the same time. To accomplish this objective, the sphere has five ports. A port in the front of the sphere where the objects are placed. A port in the back where the camera can be found. Finally, three light source ports are positioned inside the sphere in an equilateral triangle configuration. Shields are placed in these three ports to avoid direct illumination in the lens, which would cause flares.
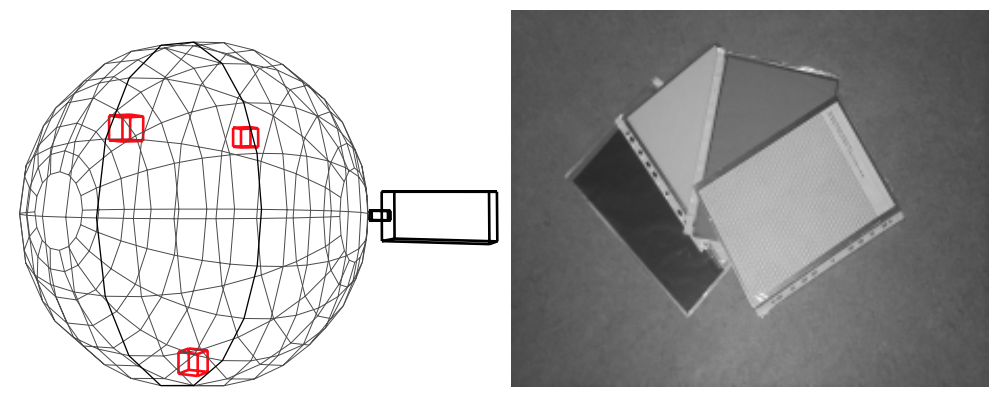

Fig. 2. Left: Light source ports. Right: the geometric and chromatic calibration sheets

Figure 2 (a) shows the position of the five ports inside the sphere. The illumination geometry obtained with this configuration creates a very homogeneous and diffuse illumination on objects placed in the output port of the sphere. The diffuse illumination eliminates specular components and for objects with regular surface removes all the shadows. However, if the object has a highly varying topology, the valid measurement region has to be constrained. 
The source of light is external to reduce the temperature in the sphere and thereby thermal noise. CCD chips work counting the number of photons that arrive at their sensor area. However, some of these photons are thermally generated. In order to obtain thermal equilibrium, the light source is turned on three hours before use. Further, during acquisition, ten images are averaged to reduce the thermal noise.

To avoid uneven intensities, a chromatic calibration is done. To achieve this, NCS sheets from Scandinavian Color Institute are selected as calibration sheets. Three NCS calibration sheets are used in the calibration: the NCS 8000, the NCS 5000 and the NCS 2000.

Once the chromatic calibration has been performed, a geometric calibration is done to correct for geometric aberration and to align the three color planes. To realize the geometric calibration a sheet with regularly spaced black disks is utilized.

Image 2 shows the geometric calibration sheet target together with the three sheets used in the chromatic calibration. The center of gravity for each of the disks is found for each channel. The centers are obtained from a threshold image using the value between the two main tops of the histogram as threshold. From the centers of gravity a grid is calculated automatically. Finally a polynomial transformation is applied to warp the centers of gravity to the grid. This warping is done on each channel separately and the parameters are found by means of a least squares fit.

After the camera is calibrated, images can be obtained. To take the images, the lesion is placed in the port in front of the sphere. To avoid the access of external light in the system, the lesion is covered with an opaque cloth.

\section{Automatic segmentation of the lesion}

Once the images have been obtained, the goal is to automatically separate the healthy and diseased areas in order to obtain statistics of the lesion. The method proposed in this work is based on the assumption that, given a suitable feature, the healthy and the diseased areas are distributed according to $\mathrm{N}\left(\mu_{1}, \sigma_{1}^{2}\right)$ and $\mathrm{N}\left(\mu_{2}, \sigma_{2}^{2}\right)$, respectively. Under this assumption, a logical criteria to separate both areas is to estimate the parameters of the Gaussians and perform a discriminant analysis. The full procedure is described step by step in the next sections.

\subsection{Removing the background}

In order to avoid external light, an opaque cloth covers the limb. This implies that the background can be seen in some images. To extract the lesion, it is necessary to eliminate this background because these pixels values can make the estimation of the parameters of the Gaussians imprecise in the next step. To accomplish this task, two facts are taking into account: the accurate calibration of the equipment and that the image background is the opaque cloth used to avoid external light. 
On one hand, the color of the cloth can be selected by looking for an object with big difference to the skin color. On the other hand, the equipment is calibrated to obtain measures that are accurate and time invariant. This means that, for a fixed band, the distribution of the background is similar in all images. These two facts together suggest using to a fixed thresholding to segment the background. In this work, the color of the cloth is chosen to be green. The reason for this is to have a good discrimination between the skin and the cloth in the red band. The value for the fixed threshold is obtained by estimating the distribution of the background in the red band for a few images.

\subsection{Finding a suitable feature}

In order to select a good feature to identify the diseased area, an exploratory analysis is conducted. The objective of this analysis is to decide which variables will make up the feature to discriminate the healthy area. Initially, a set of possible good features is selected, that is to say, the tricromatic variables and logical combinations of these such as ratios or products. To identify the features with largest discriminatory power, diseased and healthy areas are extracted from a set of images. The values of the variables for the pixels of these areas are obtained and an additional variable is added to indicate the source (healthy or diseased). A linear stepwise discriminant analysis is conducted using these values as input. The output of the discriminant analysis is the pursued feature.

\subsection{Estimating the mean and removing the healthy area}

The underlying idea to isolate the lesion is that the distribution of the obtained feature can be approximated by a linear mixture of two Gaussians. These two Gaussians correspond to the healthy and diseased distributions. The estimates of their means and variances makes it possible to identify the lesion via quadratic discriminant analysis. In this study, it has been decided to use equal prior probabilities.

To estimate the parameters of the gaussians, the distribution of the previously obtained feature is modeled by

$$
f(x)=(1-\pi) \cdot N\left(\mu_{1}, \sigma_{1}^{2}\right)+\pi \cdot N\left(\mu_{2}, \sigma_{2}^{2}\right)
$$

where $x$ is the feature vector, $\mu_{1}, \mu_{2}$ are the means and $\sigma_{1}^{2}, \sigma_{2}^{2}$ the variances (or covariance matrices if the feature is multidimensional).

The parameter are estimated iteratively via EM equations obtained setting the partial derivatives of the log likelihood equal to zero and solving with respect to the parameters involved, Taxt [8]. The algorithm is initialized using K-means, Hastie [10].

\section{Experiment}

An experiment, in collaboration with the Danish hospital of Gentofte, is conducted to test the goodness of the developed system. The goal is the acquisition 
of reproducible dermatological images and the automatic extraction of the lesions for subsequent analysis. A collection of 175 images are taken of three patients with psoriasis. The images are taken of three different areas of each patient.

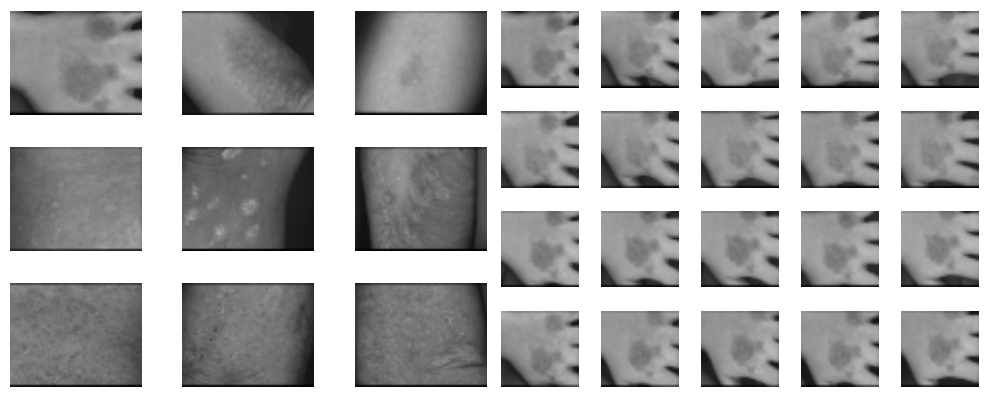

Fig. 3. Left: An example of each lesion. Right: Temporal series. Rows 1-4=week 1-4. Columns $1-5=$ repetition $1-5$.

Figure 3 left displays an example of each of the 9 different lesions taken. For each lesion, a total of twenty images are taken. These images are taken in 4 different sessions with the purpose of observing the evolution of the disease. Five images are collected per patient and session. Figure 3 right shows the obtained temporal series for one of the lesion.

It can be noticed from these images that psoriasis is characterized by silvery scales on thick red areas. Doctors score the redness, the thickness and the scaling to summarize the lesion.

In order to apply the procedure to extract the diseased area, the first step is to remove the background from the different images. Examining the histogram for the red band in a small set of images, the fixed threshold is set to 0.35. The full set of images is successfully segmented using this value for the image range from 0 to 1 .

Figure 4 left shows the removed background from the nine images. It can be noticed that no area has been removed from the two images that contain no background.

With the goal of extracting the lesion, Hance [5] and Umbaugh [6] proposed a a color space where the lesion is enhanced. The method consisted of transforming the original RGB data into a spherical domain represented by two angles and a one dimensional intensity space. They applied the transformation successful in skin tumor images. However, this transformation was not fully successful with the images of psoriasis. Patterns that explain a dermatological disease does not necessarily do so it with others.

In order to choose a suitable variable to separate the lesion in the psoriasis disease, six features comprising the trichromatic values and their logarithms are considered. Several regions are extracted from a subset of the image pool with 

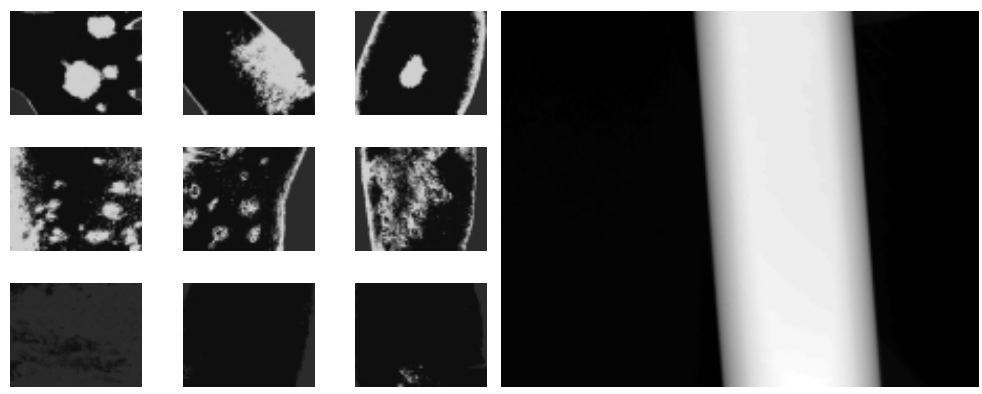

Fig. 4. Left: Segmentation of the lesion in the 9 test Images. Right: An uniform white candle

the objective of finding the goodness of the variables. The values of the considered variables correctly labeled are introduced in a linear stepwise discriminant analysis. The best combination achieved takes into account the six features. No features are discarded by the stepwise discriminant analysis procedure. However, the increment of the error is quite minimal (less than one per cent) when just the green and the blue bands are taken into account. To facilitate the comparison of the results only these two variables are considered to make up the feature.

Linear discriminant analysis is applied individually for a set of training images. The diseased and normal skin areas are labeled in these images. Just the blue and green values are introduced in the discriminant. Empirical results suggest that the best linear combination to discriminate the data is given by (green-blue).

Figure 4 Left shows the images after the healthy skin has been discarded using (green - blue).

It is noticed that some areas near the border with the background is not removed. As it has been pointed out before the integrated sphere creates a plane of diffuse light in the front port of the sphere. Only images of objects that lie in this plane are taken correctly. However, due to the curvature of most of human limbs, some parts are not in the plane so the requirement is not fulfilled and shadows appear.

To show the effect of the distance with respect to the correct plane the picture of a uniform white candle is taken. Figure 4 displays the picture taken, it can be appreciated that in the middle of the candle colors are uniform. However, as the gradient increases, the intensities go down. This decrease of the intensities in these areas means that the classifier will classify them as diseased skin. However, these shadows can be removed easily by looking at the intensities. 


\section{Summary and conclusion}

In this work, a system to take reproducible images of dermatological lesions is proposed. The system is designed to acquire geometrically and chromatically corrected images and automatically extract the lesion. The developed system is the first step in order to automatically score dermatological images. The areas identified as lesions can be summarized by statistical or pattern recognition methods.

\section{References}

1. Engstrom N., Hansson F. , Hellgren L., Tomas J., Nordin B. Vincent J. and Wahlberg A. Computerized Wound Image Analysis In Pathogenesis of Wound and Biomaterial-Associated Infections. Springer-Verlag p 189-193, 1990.

2. Hansen G., Sparrow E., Kokate J., Leland K., Iaizzo P. Wound Status Evaluation using Color Image Processing IEEE Transactions on Medical Imaging, vol. 16, no.1, February 1997

3. Marszalec E. Pietikainen M. Online Color Camera Calibration Proceedings of the 12th IAPR Internacional Conference in Pattern Recognition,vol. 1

4. Gutenev A., Skladnev V.N, Varvel D. Acquisition-time image quality control in digital dermatoscopy of skin lesion. Computerized Medical Imaging and graphics 25 (2001) 495-499

5. Hance G., Umbaugh S., Moss R., Stoecker W. Unsupervised Color Image Segmentation IEEE Engineering in Medicine and Biology, February 1996

6. Umbaugh S, Moss R. Automatic Color Segmentation of Images with Application to Detection of Variegated Coloring in Skin Tumors IEEE Engineering in Medicine and Biology, December 1989

7. Johnson R., Wichern D.,: Applied Multivariated Statistical Analysis. Prentince-Hall, Berlin Heidelberg New York (1995)

8. Taxt T., Hjort L., Eikvik L. : Statistical Classification using a Linear Mixture of two Multinormal Probability Densities. Pattern Recognition Letters.(1991) 12 731-737

9. Folm-Hansen R.: On Chromatic and Geometrical Calibration. PhD tesis ,Lyngby (1999)

10. Hastie T., Tibshirani, R., Friedmamn, J.: The Elements of Statistical Learning. Springer 